\title{
Online Parametric Identification of Power Impedances to improve Stability and Accuracy of Power-Hardware-in-the-Loop Simulations
}

\author{
Antonino Riccobono, Member, IEEE, Eyke Liegmann, Student Member, IEEE, Marco Pau, Member, IEEE, \\ Ferdinanda Ponci, Senior Member, IEEE and Antonello Monti, Senior Member, IEEE
}

\begin{abstract}
This paper presents the Wideband System Identification (WSI) technique, i.e. an online method to identify power impedances over a wide frequency range from which the corresponding parametric impedance can be calculated online as well. The WSI technique exploits an existing custom $25 \mathrm{~kW}$ power electronic converter on the top of its power conversion function, which serves as power amplifier of an existing Power Hardware in the Loop (PHiL) simulation setup. The PHiL simulation technique allows connecting a real Device Under Test (DUT) with the realtime simulated Rest Of System (ROS) at power level. An Interface Algorithm (IA) on simulation side and a power amplifier (the 25 kW power electronic converter) connect ROS and DUT. The paper shows the impact of the uncertainties in the WSI chain on the accuracy of the impedance identification and highlights how the WSI technique can be combined with the Damping Impedance Method (DIM) IA to improve both accuracy and stability of the PHiL test bench. The application of the method is illustrated for the scenario of a PHiL test of a DC microgrid with a passive load.
\end{abstract}

Index Terms-impedance measurement; wideband impedance identification; power hardware in the loop; measurement uncertainty;

\section{NOMENCLATURE}

$\begin{array}{ll}\text { ADC } & \text { Analog to Digital Converter } \\ \text { DFT } & \text { Discrete Fourier Transform } \\ \text { DIM } & \text { Damping Impedance Method } \\ \text { DMA } & \text { Direct Memory Access } \\ \text { DUT } & \text { Device Under Test } \\ \text { FIFO } & \text { First In First Out } \\ \text { FlePS } & \text { Flexible Power Simulator } \\ \text { FPGA } & \text { Field Programmable Gate Array } \\ \text { IA } & \text { Interface Algorithm } \\ \text { IGBT } & \text { Insulated Gate Bipolar Transistor } \\ \text { NCS } & \text { Naturally Coupled System } \\ \text { PHiL } & \text { Power Hardware in the Loop }\end{array}$

A. Riccobono, E. Liegmann, M. Pau, F. Ponci and A. Monti are with the Institute for Automation of Complex Power Systems, RWTH Aachen University, Aachen 52062, Germany (email: [ariccobono, eliegmann, mpau, fponci, amonti]@eonerc.rwth-aachen.de).

(C) 2017 IEEE. Personal use of this material is permitted. Permission from IEEE must be obtained for all other uses.

DOI: 10.1109/TIM.2017.2706458.

Publisher version: http://ieeexplore.ieee.org/abstract/document/7955003/
PRBS Pseudo Random Binary Sequence

RMSE Root Mean Square Error

ROS Rest Of System

RTDS Real-Time Digital Simulator

WSI Wideband System Identification

\section{INTRODUCTION}

In recent years, there has been an increasing interest in methods for wideband identification of power impedances [1][12]. These methods consist in generating a wideband perturbation controlled in both magnitude and duration for both voltage and current at the impedance measurement point. Then, advanced digital signal processing techniques can be applied on the measured voltage and current to identify the impedance of the system under test. Some of proposed solutions in the literature use dedicated power hardware (such as extra electronic amplifier or switching passive loads) to excite the wideband perturbation at power level which are recognized to be invasive methods [8]-[10], while some others use existing power hardware (such as existing power electronic converters or switching passive loads) which are recognized to be noninvasive methods [1]-[7],[11]-[12]. Among the noninvasive solutions, methods for wideband identification of power impedances in conjunction with existing power electronics converters are quite attractive, especially in modern power electronic distribution systems [2]-[7].

This paper presents a noninvasive online wideband impedance identification technique, called the Wideband System Identification (WSI) technique. It consists of injecting a short-time small-signal approximation of white noise (wide bandwidth in nature), i.e. Pseudo Random Binary Sequence (PRBS), on the control of an existing power converter while it is performing its power conversion function. Then, digital processing is performed in a dedicated control platform to identify the impedance of the connected device from the voltage and current measurements over the length of the injection. With respect to previous publications in the field of wideband measurement of power impedances [2]-[4], [8]-[12], the present work includes the online parametrization of the identified nonparametric impedance in the same control platform. The parametrization of the identified impedance is the key enabler 
for several system-level applications, such as system health monitoring, harmonic propagation detection, active filter monitoring, and adaptive control [1]-[12]. As a special application of the WSI technique, this paper presents how this type of measurement can improve the stability and accuracy of an existing Power Hardware in the Loop (PHiL) simulation setup.

The PHiL simulation technique allows testing a physical Device Under Test (DUT) connected to the real time simulated Rest Of System (ROS) through an Interface Algorithm (IA) and a power amplifier. This technique has been increasingly used by power system engineers to test power hardware [16]-[17], such as loads, converters, generators and protective devices, connected to complex power system models running in a realtime digital simulator. Classical advantages of PHiL simulation techniques lay on the reduction of costs, risk, and time associated with each design cycle, and the increase of the safety of laboratory personnel. Despites their unquestionable advantages, PHiL setups present challenges in terms of stability and accuracy, mainly due to the non-idealities of the power amplifier [18]. These non-idealities, e.g. delays, sensing errors, non-linearity, and limited bandwidth, prevent the power amplifier from tracking the reference accurately over a wide frequency band. The IA plays a key role to compensate for the shortcomings of the power amplifier and improves both the stability and the accuracy of PHiL simulations. Several IAs are investigated and compared in [18]. Among them, the Damping Impedance Method (DIM) IA "is recommended for most PHiL applications" [18], as it offers the best tradeoff between stability and accuracy. However, this method requires knowledge of the impedance of the DUT. The WSI technique is suggested in [19]-[21] as a method to identify the DUT impedance and it is here integrated in an existing PHiL simulation setup to improve its stability and accuracy.

This paper extends previously presented work [7] by investigating the impact of the uncertainty components present in the chain of the WSI technique. In particular, it is shown how the uncertainty characteristics of the WSI tool and the resulting accuracy of the parametric impedance can play a key role for the stability of PHiL test benches. Moreover, the benefits of the WSI to improve the accuracy and stability of an existing PHiL setup are presented and discussed in detail.

\section{THE WIDEBAND System IDENTIFICATION TeCHNIQUE}

This section presents the online WSI technique exploiting the custom $25 \mathrm{~kW}$ power electronic converter, i.e. the power amplifier of the PHiL setup called Flexible Power Simulator (FlePS) (fully described in Section III). The peculiarity of this technique is in how the perturbation is realized. Instead of performing a small-signal frequency sweep which requires long injection times, a short-time small-signal PRBS is used as test signal. PRBS is a digital approximation of white noise, therefore wideband in nature, and this allows creating a wideband perturbation at the impedance measurement point within a time window of $\mathrm{ms}$.

Fig. 1 shows the conceptual block diagram of the implemented WSI technique. In practice, small-signal PRBS is

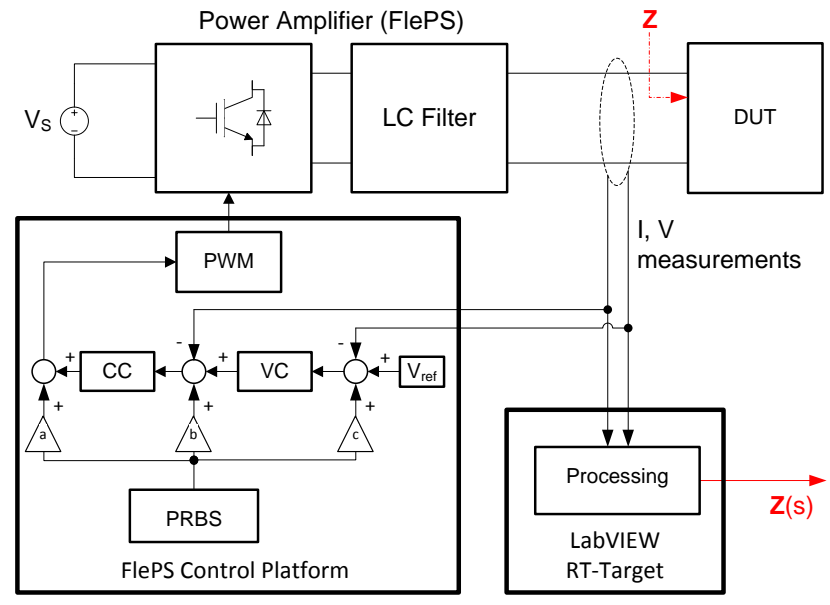

Fig. 1. Block diagram of WSI technique.

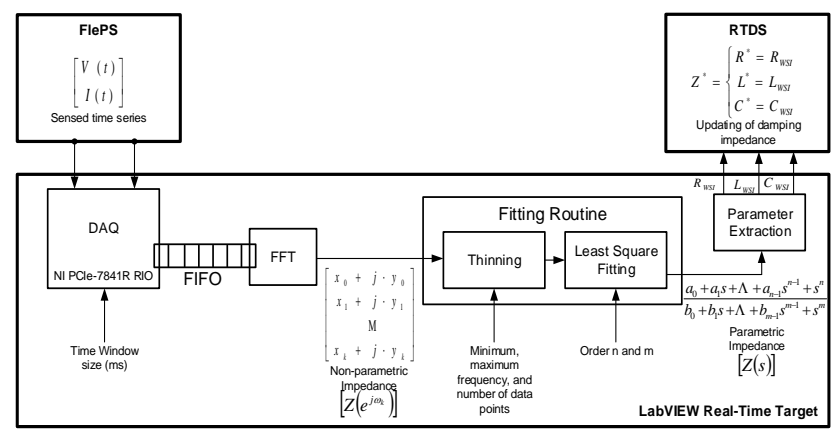

Fig. 2. Block diagram of the post processing routine to identify the parametric impedance.

added to the duty cycle and to both the current and voltage reference signals of the FlePS controller. The reason for such a choice is linked to the frequency response of the closed-loop converter. If PRBS is injected to the duty cycle only, PRBS, seen as a disturbance, is rejected within the outer control bandwidth by effect of the nested control loops, while it is not rejected beyond the bandwidth of the outer control loop. On the other hand, if PRBS is injected to the current and voltage reference signals, it is not rejected within the current and voltage control bandwidths. Therefore, injecting PRBS to the duty cycle and to both the current and voltage reference signals ensures that PRBS is not rejected by the control action over a wide frequency range. Reference [6] contains some more details about the effect of the control loop to PRBS. Notice from Fig. 1 that, to obtain the desired controlled perturbation amplitude at the impedance measurement point, the PRBS signal needs to be properly scaled by the factors "a", "b", and "c" before being added to the duty cycle, current reference, and voltage reference, respectively.

The operating point of the DUT remains the same during the injection as the PRBS is a zero mean process and each value is uncorrelated with all the other values in the sequence [1]-[6]. Then, voltage and current measurements are processed in a dedicated LabVIEW Real-Time PC (RT-Target) that includes a NI RIO board (PCIe-7851R) to identify the parametric impedance of the connected DUT.

Fig. 2 gives a schematic representation of the implemented 
WSI technique. Notice that PRBS is generated within FlePS control platform as highlighted in Fig. 1. While the DUT is being perturbed, voltage and current at the terminals of the DUT are sampled with rate of up to $750 \mathrm{kS} / \mathrm{s}$ thanks the 16-bit Analog to Digital Converter (ADC) of the NI RIO board. The embedded FPGA (Virtex5) allows buffering the sampled values in a FIFO through a Direct Memory Access (DMA) channel. The subsequent processing routines are implemented on the CPU of the LabVIEW RT-Target. It is proven in [1]-[2] that the impedance of the DUT can be determined by dividing the Discrete Fourier Transform (DFT) of voltage $v[n]$ and current $i[n]$ samples.

$$
Z\left[e^{j \omega}\right]=\frac{D F T\{v[n]\}}{D F T\{i[n]\}}
$$

This yields the non-parametric impedance consisting of $\mathrm{N}$ complex data points:

$$
N=t_{\text {window }} \cdot f_{\text {sampling }}
$$

where $t_{\text {window }}$ is the observation window during which PRBS is injected and voltage and current are measured. Then, the fitting routine online calculates the parametric transfer function from the non-parametric impedance. To impose equal fitting priority across all frequencies on the non-parametric data, a thinning technique is used to obtain a logarithmically spaced subset of the data points. This method reduces the computational time of the numerical least square fitting algorithm, which calculates the coefficients of the parametric impedance $Z(s)$. The least square fitting requires as input the order of the numerator $n$ and denominator $m$. Finally, the proper parameters in terms of $R_{W S I}, L_{W S I}$, and $C_{W S I}$ are extracted from the parametric transfer function $Z(s)$, and they will update the damping impedance (this latter step is explained in detail in Sections III and IV).

The execution time of the complete identification consists of the chosen time window and the impedance calculation time. The latter comprises the execution time of the DFT algorithm, which is of complexity $O(N \cdot \log (N))$, and of the fitting routine, which depends on exit conditions of the least-square fitting algorithm [25].

\section{A. Overcoming Practical Challenges}

A major practical challenge in impedance identification consists of maintaining the small-signal condition and, at the same time, guaranteeing a measurable perturbation within the frequency range of interest. In theory, the maximum identifiable frequency is defined by the Nyquist frequency [1][6], i.e. half the switching frequency of the power converter FlePS. In practice, the upper boundary depends on the amplitude of the injected PRBS signal. In fact, the output filter of the power converter attenuates the high frequency perturbation, eventually leading to a signal level that is comparable with the noise floor $(<-60 d B)$ and therefore too small to be identified [1]. The lower frequency boundary, instead, is equal to the inverse of the measured time window $t_{\text {window }}$ during which the PRBS is injected. As a consequence, the attenuation at high frequencies introduced by the output filter dictates the minimum amplitude of the injected PRBS signal. Therefore, to obtain a good identification as close as possible up to the Nyquist frequency, despite the attenuation of the output filter, and maintaining at the same time the smallsignal condition, a good choice is to perturb voltage and current with magnitude between $5 \%$ and $10 \%$ of their steady-state value. As alternative to white noise, blue noise, which has a larger magnitude at high frequencies without affecting the amplitude at low frequencies, can be used. A blue noise filter can be applied to white noise PRBS to obtain a blue noise injection [2]-[3].

The superimposed perturbation signal has small amplitude with respect to the steady state voltage and current supplying the DUT and, therefore, likely to be noisy especially at high frequencies as explained before. Consequently, the ADC, necessary to measure voltage and current, plays also a key role for accurate identification. The chosen NI RIO board is equipped with a 16-bit ADC, which allows achieving a resolution of $0.305 \mathrm{mV}$ on the maximum input analog voltage range of $\pm 10 \mathrm{~V}$. The minimum measurable voltage perturbation amplitude is given by $0.305 / 0.01185=25.74 \mathrm{mV}$, where 0.01185 is the voltage transducer gain. The minimum measurable current perturbation amplitude is given by 0.305 / $0.8=0.38 \mathrm{~mA}$, where $0.8 \mathrm{~V} / \mathrm{A}$ is the current transducer gain.

The choice of the time window is another practical challenge worthy of discussion. As the time window is directly linked to the number $\mathrm{N}$ of complex data points of the non-parametric impedance according to (2), for a fixed sampling rate, a long enough time window should be selected in order to have enough data points to capture eventual sharp features of the identified impedance, such as lightly damped resonances. On the other hand, the time window should be chosen short enough to avoid increasing impedance calculation times. Moreover, how short the time window should be chosen is in relation to the characteristics of the DUT. For active DUTs, such as power electronic converters, it is important to set the time window short enough in order to catch with fast changing impedances for example due to load steps. For highly nonlinear DTUs, such as diode bridges, reference [9] points out the need for a time window equal to half cycle of the nominal system frequency. This paper considers only passive power impedances as DUT and a practical choice is $t_{\text {window }}=100 \mathrm{~ms}$.

Another challenge is given when testing active systems with harmonic emission. By referring, for example, to the identification of Y-connected grid impedance described in [6], the presence of the fundamental frequency $(50 \mathrm{~Hz})$ on the active system under test (the grid itself) affects the accuracy of the non-parametric impedance identification around the system fundamental frequency, as depicted in Fig. 8 of [6], which shows three data points off around $50 \mathrm{~Hz}$. To correct them, the voltage and current spectra acquired with and without PRBS, shown in Figs. 3-4, are subtracted as done in [6]. The corrected voltage and current spectra are depicted in Fig. 5, which shows the cancellation of the $50 \mathrm{~Hz}$ component. Since the cancellation is never perfect, it is proven in [6] that it is possible to improve the accuracy of the non-parametric impedance measurement by applying windowing (Hanning) to the DFT and performing synchronous acquisitions to ensure periodicity of the signal being processed by the DFT. The corrected non-parametric 

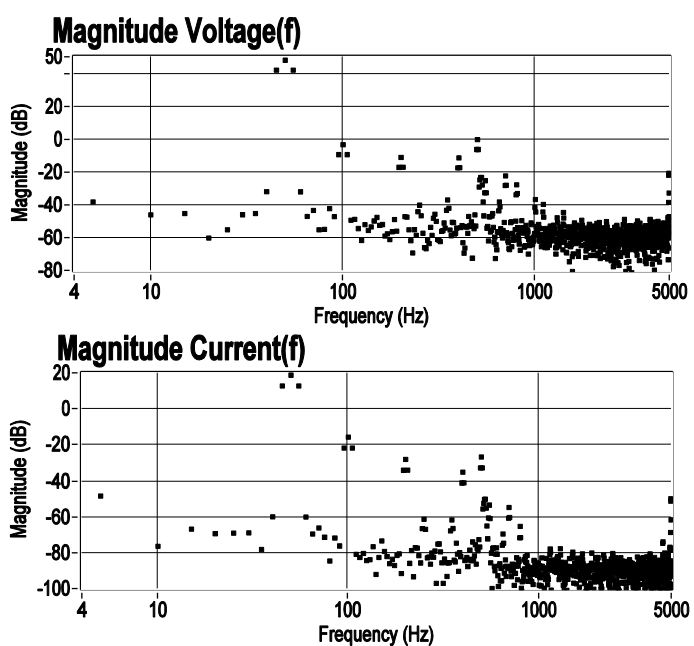

Fig. 3. Voltage and current spectra without PRBS for the identification of Y-connected active impedance in [6].
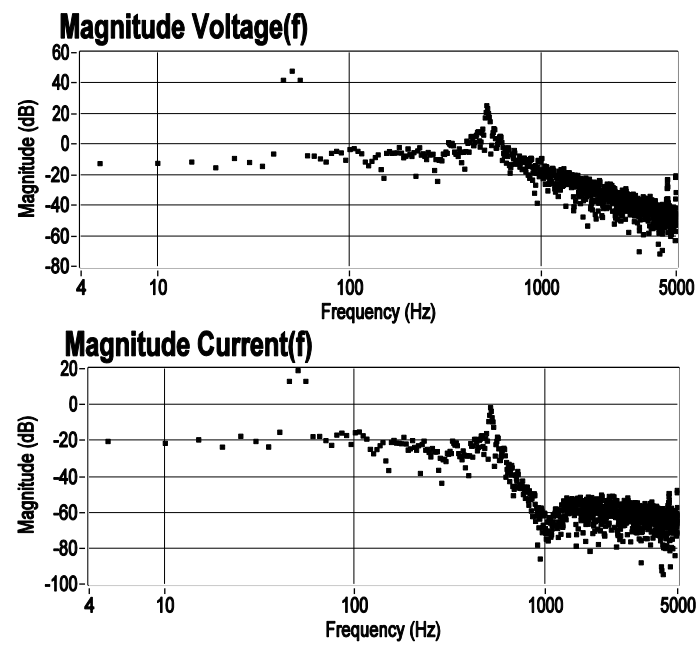

Fig. 4. Uncorrected voltage and current spectra with PRBS for the identification of Y-connected active impedance in [6].
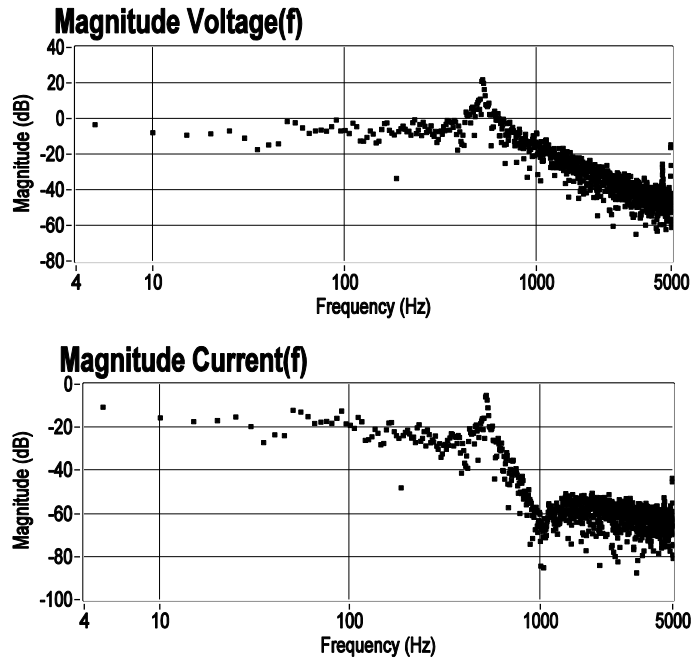

Fig. 5. Corrected voltage and current spectra without PRBS for the identification of Y-connected active impedance in [6]. impedance identification is depicted in Fig. 9 of [6], which shows that the three data points around $50 \mathrm{~Hz}$ have been reduced of about $20 \mathrm{~dB}$ in magnitude, while there is not significant improvement in phase. As the focus of this paper is the identification of passive impedances, the problem of existing harmonics is not present.

\section{B. Wideband System Identification Uncertainty}

As it will be shown in Section V, the reliable operation of the PHiL simulations can be strongly affected by the accuracy of the identified impedance parameters. For this reason, it is important to analyze the WSI chain and to estimate the uncertainty resulting due to the different uncertainty contributions. The computation of this uncertainty budget in analytical way is not easy because several hardware and software components (each one with its own uncertainty contribution) are in the WSI chain, and because a detailed characterization in the frequency domain of all these components would be needed. Last but not least, the random nature of the injected PRBS signal does not allow any a priori knowledge of the measured quantities characteristics, thus further complicating the scenario. The following uncertainty components affect the final WSI impedance results:

1) Sensors uncertainties: random errors given by current and voltage sensors are the first cause of uncertainty in the WSI chain; it is worth to remark that, because of the added white noise, both voltage and current have multiple tones which can be characterized by different uncertainties (depending on the sensor characteristics) both in magnitude and phaseangle.

2) $A D C$ uncertainties: as known in the literature (see for example [15]), the analog to digital conversion stage is responsible of uncertainties due to the number of bits used in the quantization process, the time jitter and the microprocessor wordlength. Also in this case, a different impact can result for the tones at different frequencies and for magnitude and phase-angles.

3) DFT uncertainties: the DFT algorithm is not only responsible for the propagation of the uncertainties to the spectrum components, but it also brings additional uncertainties due to the leakage of inter-harmonic components. Windowing solutions can be used to mitigate the problem, but the random nature of the PRBS signals prevents the use of more advanced techniques to fully compensate for leakage errors.

4) Impedance calculation: as shown in (1), the non-parametric impedance is obtained through the division of voltage and current spectrum components. This step leads to the propagation of the uncertainties present in the voltage and current tones to the corresponding impedance components.

5) Least Square Fitting: an additional uncertainty contribution is brought by the iterative algorithm used to fit the impedance tones to the parametric model assumed for the impedance. Here both the fitting routine and the chosen model are sources of uncertainties.

6) Impedance parameters calculation: the resulting uncertainties of the coefficients of the transfer function (obtained as output from the fitting algorithm) are eventually propagated to the final impedance parameters. 


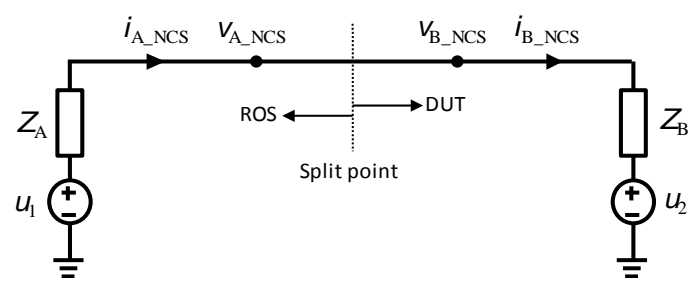

(a)

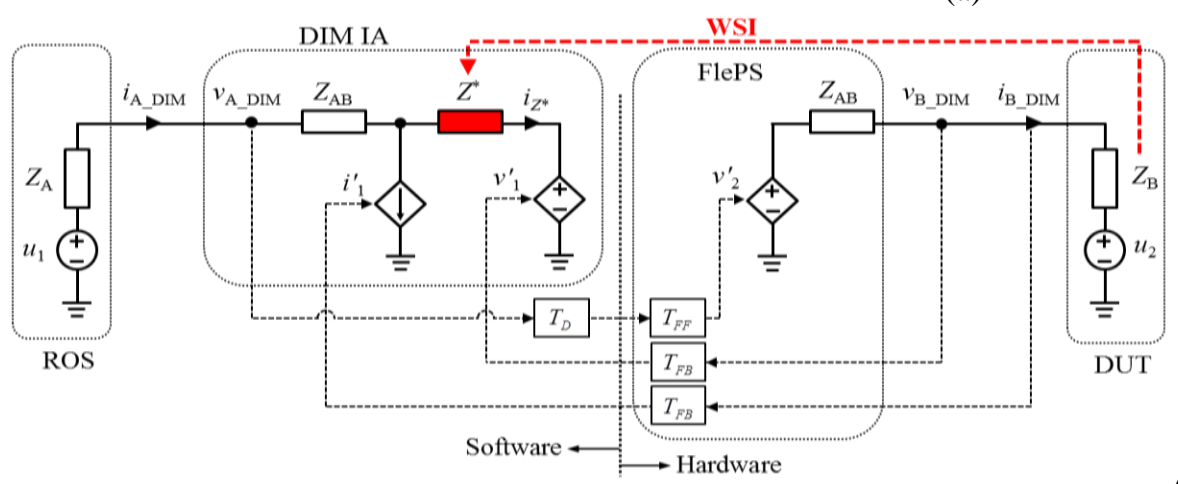

(b)

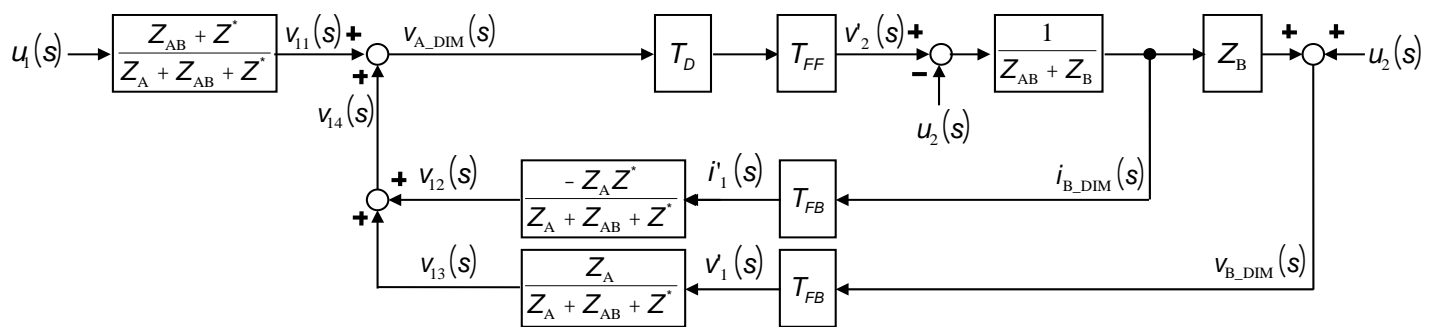

(c)

Fig. 6. (a) Voltage divider as NCS; (b) correspondent PHiL setup with MDIM IA; (c) equivalent block diagram of PHiL system with DIM IA.

\section{POWER HARDWARE IN THE LOOP - INTERFACE ALGORITHM}

This section presents the PHiL implementation with DIM IA and explains the motivation for identifying the DUT impedance. The presented scenario consists of a PHiL setup emulating a voltage divider, i.e. the Naturally Coupled System (NCS), as shown in Fig. 6(a). In this representation, the voltage source $u_{1}$ and the impedance $Z_{\mathrm{A}}$ are part of the ROS, while the voltage source $u_{2}$ and the impedance $Z_{B}$ are part of the DUT. To build the PHiL system, the NCS is split into the ROS and the DUT. Fig. 6(b) shows the same system as PHiL setup with DIM IA. The power amplifier is modeled as a voltage-controlled voltage source with the feed forward transfer function $T_{F F}$, which models the amplifier limited bandwidth, and impedance $Z_{\mathrm{AB}}$, which models the amplifier output impedance. The voltage reference $v_{\text {A_DIM }}$ is sent from the real-time simulator to the power amplifier. At the same time, current $i_{\text {B_DIM }}$ and voltage $v_{\mathrm{B} \_ \text {DIM }}$ are sensed and fed back to be included in the real-time simulation. The transfer functions $T_{\mathrm{D}}$ and $T_{\mathrm{FB}}$ model the time delay introduced by the real-time simulator and the sensing gain, respectively. The impedance $Z^{*}$ is the damping impedance of the DIM IA. The open loop transfer function of the PHIL system with DIM IA (G $\mathrm{G}_{\text {OL DIM }}$ ) is derived from the equivalent block diagram shown in Fig. 6(c) [20]:

$$
G_{O L D I M}=\frac{Z_{A}\left(Z_{B}-Z^{*}\right)}{\left(Z_{a b}+Z_{B}\right)\left(Z_{A}+Z_{a b}+Z^{*}\right)} T_{D} T_{F F} T_{F B}
$$

which shows that, if the following condition is achieved,

$$
Z^{*}=Z_{B}
$$

$\mathrm{G}_{\text {OL DIM }}$ becomes zero and, therefore, never crosses the -1 point in the Nyquist diagram, thus, guaranteeing absolute stability.

As done in [20]-[21], considerations about the accuracy of the PHiL system can be formulated, by evaluating voltages and currents of the PHiL system with DIM IA on the simulation side ("A" subscript) and on the DUT side ("B" subscript) compared to those of the NCS picked as test case reference. The equations in Table I are derived by inspection of Fig. 6(a) for the NCS and of Fig. 6(c) for the PHiL system for the simpler case when $\mathrm{u}_{2}=$ 0 . The accuracy improvements of the PHiL system with DIM IA are evident when the following conditions are both true:

$$
Z_{A B}=0 \text { and } Z^{*}=Z_{B}
$$

The right column of Table I shows that the voltage and current at the ROS side perfectly match those ones of the NCS, while the performance at the DUT side is mainly determined by the bandwidth of the power amplifier. The considerations above about stability and accuracy improvements of PHiL experiments with DIM IA lead to the need to precisely identify the impedance of the DUT through the WSI tool presented in Section II. The Damping Impedance $Z^{*}$ can be updated while 
the real-time simulation is running (see Fig. 6(b) in red), so that stability and accuracy of the PHiL system are guaranteed over a wide range of operating conditions.

TABLE I.

\begin{tabular}{|c|c|c|}
\hline NCS & PHiL: DIM IA & $\begin{array}{c}\text { PHiL: MDIM IA } \\
\text { when } \\
Z_{\mathrm{AB}}=0, Z^{*}=Z_{\mathrm{B}}\end{array}$ \\
\hline $\begin{array}{l}v_{A_{-} N C S} \\
=\frac{Z_{B} \cdot u_{1}}{Z_{A}+Z_{B}}\end{array}$ & $v_{\mathrm{A}_{-} \mathrm{DM}}=\frac{\frac{Z_{A B}+Z^{*}}{Z_{A}+Z_{A B}+Z^{*}} u_{1}}{1-\frac{Z_{A B}+Z^{*}}{Z_{A}+Z_{A B}+Z^{*}} \cdot T_{F B} T_{F F} T_{D}\left(1-\frac{Z^{*}}{Z_{B}}\right)}$ & $\begin{array}{l}v_{A_{-} M D I M} \\
=\frac{Z_{B}}{Z_{A}+Z_{B}} u_{1} \\
=v_{A_{-} N C S}\end{array}$ \\
\hline $\begin{array}{l}v_{B_{-} N C S} \\
=v_{A_{-} N C S}\end{array}$ & $v_{\mathrm{B} \_\mathrm{DM}}=T_{F F} T_{D} v_{\mathrm{A} \_\mathrm{DIM}}$ & $\begin{array}{l}v_{B_{-} D I M} \\
=T_{F F} T_{D} \cdot v_{A_{-} N C S}\end{array}$ \\
\hline $\begin{array}{l}\overline{i_{A_{-} N C S}} \\
=\frac{u_{1}}{Z_{A}+Z_{B}}\end{array}$ & $i_{\mathrm{A}_{-} \mathrm{DIM}}=\frac{u_{1}-v_{A_{-} D M}}{Z_{\mathrm{A}}}$ & $\begin{array}{l}i_{A_{-} D I M}=\frac{u_{1}}{Z_{A}+Z_{B}} \\
=i_{A_{-} N C S}\end{array}$ \\
\hline $\begin{array}{l}i_{A_{-N N C S}} \\
=i_{B_{-} N C S}\end{array}$ & $i_{\mathrm{B}_{-} \mathrm{DIM}}=\frac{1}{Z_{A B}+Z_{B}} T_{F F} T_{D} v_{\mathrm{A}_{-} \mathrm{DM}}$ & $\begin{array}{l}i_{B_{-} D I M} \\
=T_{F F} T_{D} \cdot i_{A_{-} N C S}\end{array}$ \\
\hline
\end{tabular}

\section{EXPERIMENTAL SETUP}

The experimental setup of the PHiL test bench is depicted in Fig. 7. It also highlights in red the addition of the WSI tool. The WSI tool performs two tasks: first, it online identifies the DUT parametric impedance, and then it sends the identified parameters to the Real-Time Digital Simulator (RTDS) where the damping impedance $Z^{*}$ is updated.

\section{A. Real-Time Digital Simulator (RTDS)}

RTDS runs the models of ROS and IA in real time. Here, the hardware and software by RTDS Technologies Inc. is utilized, with a fixed time-step of $50 \mu \mathrm{s}$. At each time step RTDS calculates the voltage reference for the power amplifier, while at the same the simulation receives the measured voltage and current from FlePS sensing unit. At the same time, the Damping Impedance $Z^{*}$ can be updated while the PHiL experiment is running.

\section{B. Power Amplifier (FlePS)}

The power amplifier of the PHiL setup is the Flexible Power Simulator (FlePS) [22]-[23]. The power section of FlePS is depicted in Fig. 8. It consists of a three-phase isolating transformer, an active front end, and a DC link. Two of the four available IGBT legs form a DC/DC full bridge topology. The LC output filter with small damping resistors is set to realize a voltage source converter. This gives the opportunity to utilize a unipolar PWM modulation, which further reduces the switching ripple by doubling the apparent switching frequency to $80 \mathrm{kHz}$. A control scheme is implemented consisting of an inner deadbeat inductor current loop with an outer voltage PI controller. The crossover frequency of the voltage loop is 500 $\mathrm{Hz}$. This means that the output of the power amplifier cannot track fast transients of the reference signal coming from RTDS.

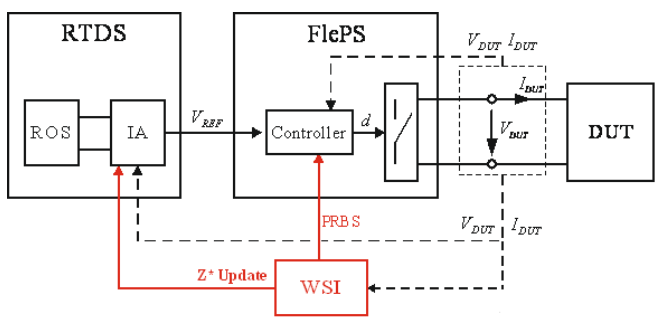

(a)

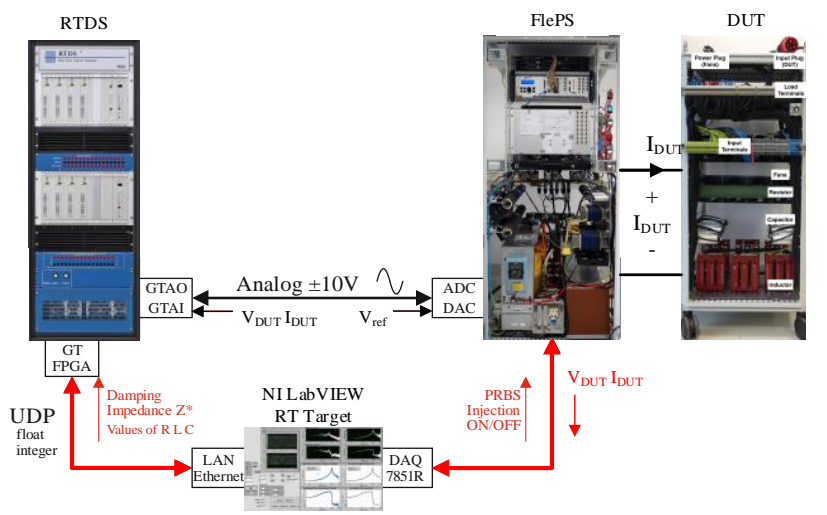

(b)

Fig. 7. Experimental setup of PHiL test bench: (a) block diagram, and (b) including the communication interfaces.

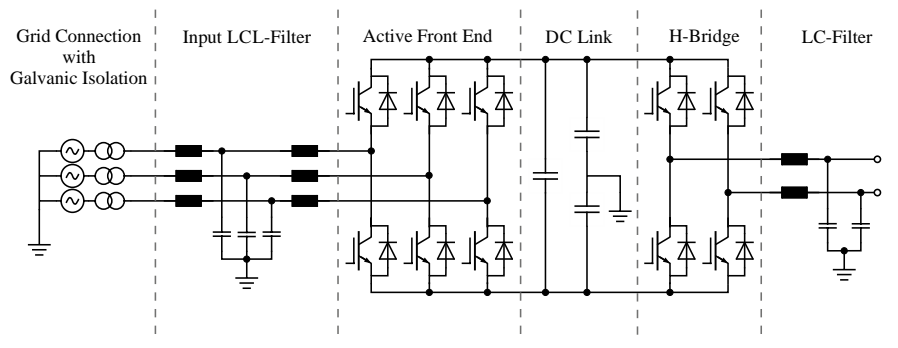

Fig. 8. Power hardware of power amplifier FlePS.

\section{Communication Interfaces}

Fig. 7(b) shows the communication interfaces of the PHiL test bench, including information about the protocol and data type, as well as showing which information is being exchanged. The analog interface between RTDS and FlePS allows the signal exchanges at the Software/Hardware interface of Fig. 6(b). The digital interface between the NI LabVIEW RT Target and FlePS allows the synchronization of the PRBS injection with the data acquisition for the WSI technique. A dedicated FPGA (GT FPGA) bridges the data exchange between the RT Target and RTDS, which is used to update the damping impedance $Z^{*}$ of the DIM IA. The identified values of the passive load, i.e. R, L, and C, are directly available in RTDS and included in the DIM IA.

\section{Device Under Test}

The DUT is a flexible passive load rack that can be connected in various configurations. Here, single-phase RL and RLC loads are chosen as depicted in Fig. 9, described by their transfer functions (6) and (7).

$$
Z_{R L}=R+s \cdot L
$$




$$
Z_{R L C}=\frac{R+s \cdot L}{1+s \cdot R C+s^{2} \cdot L C}
$$
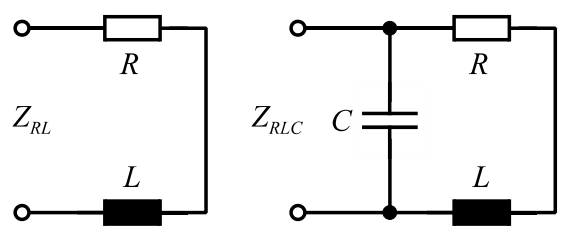

Fig. 9. Passive DUT configurations.

\section{RESULTS AND DISCUSSION}

This section presents the determination of the performance of the impedance measurement via WSI tool and the propagation of its uncertainty onto the accuracy and stability of the PHiL experimental setup.

\section{A. Wideband System Identification of DUT}

As shown in Section II, the WSI technique allows identifying the non-parametric impedance of the DUT and translating it in its parametric form. The uncertainties brought and propagated during the different steps of the WSI result in an overall uncertainty for the identified impedance parameters. While the uncertainties given by sensors and ADC can be computed analytically following an ad hoc characterization of the used hardware, it is challenging to evaluate the uncertainties arising due to the software processing. For this reason, the uncertainty effects given by DFT and least squares fitting algorithm have been assessed here through off-line simulation tests.

Fig. 10 shows the results obtained through the DFT for the non-parametric RLC DUT impedance when: 1) no uncertainty is considered in the magnitude and phase-angles of current and voltage tones, and 2) 5\% uncertainty is considered for the magnitude of each current and voltage tone of the signal. In this test, white noise is emulated generating 10000 voltage components (overlapped to the $50 \mathrm{~Hz}$ signal) with random frequency, amplitude and phase-angle and calculating the resulting current flowing through the DUT impedance. It is possible to observe that when no uncertainty is considered, the estimated impedance has a very good matching with the true one. Such result points out that the errors brought by the leakage in the DFT are in this case almost not propagated to the impedance when applying the division in (1). On the other side, when the inputs are uncertain, this compensation does not hold any more and both leakage and input signal errors are clearly propagated to the impedance.

Using the same test set-up, Table II shows the propagation of the uncertainty to the final impedance parameters, including both DFT and fitting routine (test has been performed through a Monte Carlo simulation with 1000 trials). It is possible to observe that in this case, also when no uncertainty is considered, the small errors given by the DFT are translated in non-negligible uncertainties when applying the fitting algorithm. In general, as it could be expected, the uncertainty trend is increasing for larger values of input uncertainties.

In Figs. 11-12, the non-parametric impedance (black dots) and the fitted parametric impedance (red line) obtained in the online PHiL scenario are plotted and compared with the

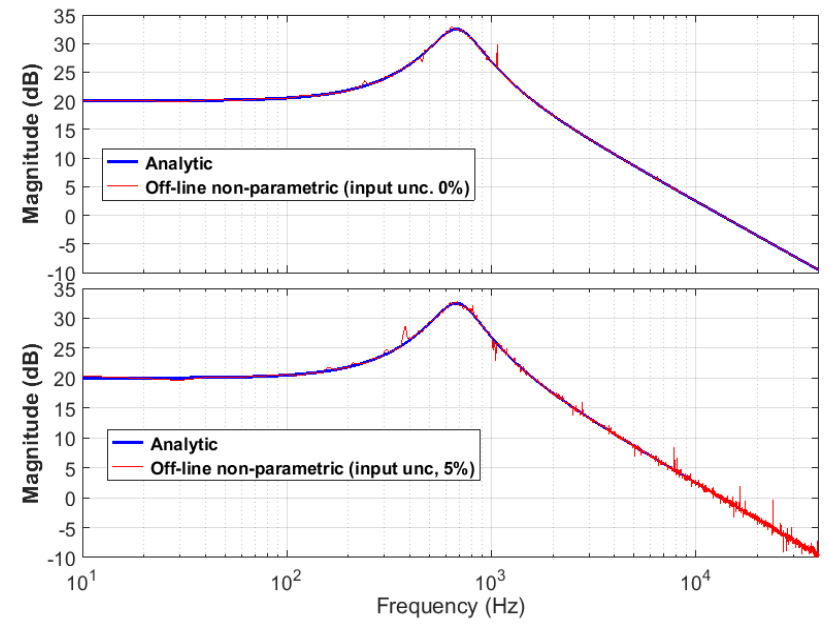

Fig. 10. Bode plot (magnitude only) of the non-parametric RLC DUT impedance (red) with different input uncertainties to the DFT compared to the analytical model (blue).

TABLE II.

PARAMETERS UNCERTAINTY WITH DIFFERENT LEVELS OF INPUT SIGNAL UNCERTAINTY

\begin{tabular}{|c|c|c|c|}
\hline \multirow{2}{*}{$\begin{array}{c}\text { Amplitude } \\
\text { uncertainty } \\
\text { input signal }\end{array}$} & \multicolumn{3}{|c|}{ \% Relative Uncertainty (30) } \\
\cline { 2 - 4 } & $\mathbf{R}$ & $\mathbf{L}$ & $\mathbf{C}$ \\
\hline $\mathbf{0 \%}$ & 3.1 & 3.4 & 3.5 \\
\hline $\mathbf{5 \%}$ & 4.5 & 4.8 & 4.5 \\
\hline $\mathbf{1 0 \%}$ & 7.5 & 7.0 & 7.3 \\
\hline
\end{tabular}

analytical transfer function of the DUT (blue line) which is determined by measuring each electrical component individually with a frequency response analyzer, i.e. a Model 350c from Venable Instruments, and applying (6) and (7). It is possible to observe that the identified non-parametric impedance presents a certain scattering of the values due to the unavoidable uncertainties coming from the upstream hardware components.

The parametric identified values have been statistically characterized through a Monte Carlo analysis (by means of

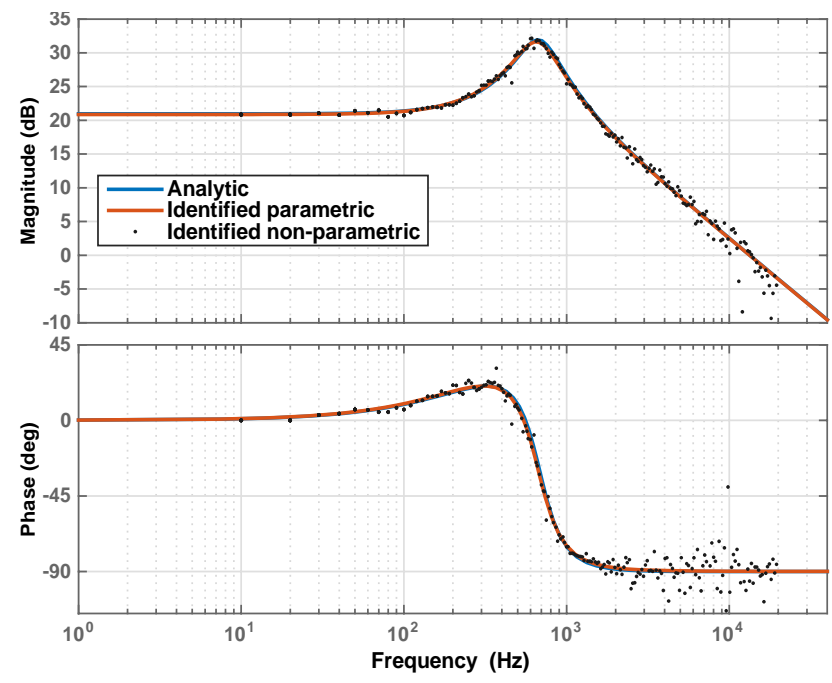

Fig. 11. Bode plot of parametric RLC DUT impedance (red) compared to the counterpart non-parametric (black) and the analytical model (blue). 

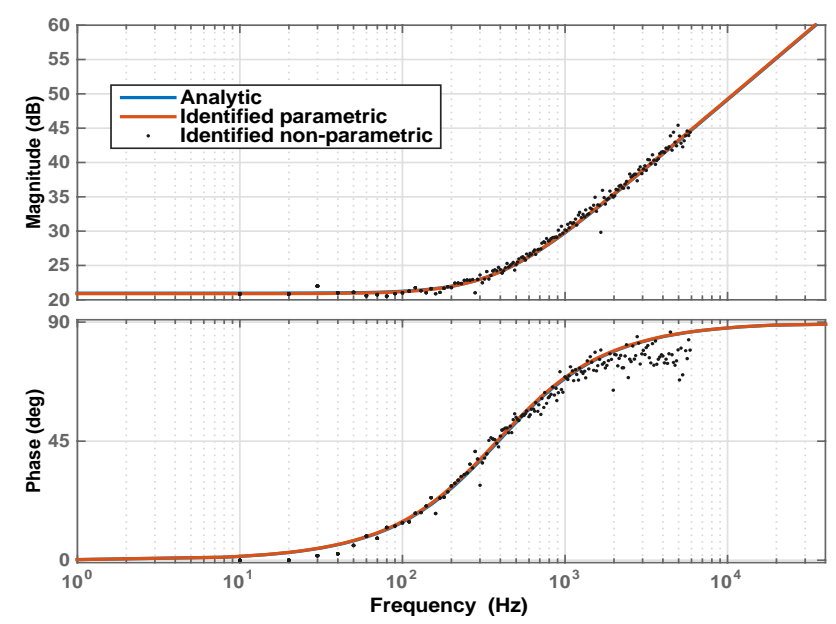

Fig. 12. Bode plot of parametric RL DUT impedance (red) compared to the counterpart non-parametric (black) and the analytical model (blue).

TABLE III.

IDENTIFIED VS. MEASURED VALUES OF RLC LOAD

\begin{tabular}{|c|l|l|l|}
\hline & \multicolumn{1}{|c|}{$\mathbf{R}$} & \multicolumn{1}{c|}{ L } & \multicolumn{1}{c|}{ C } \\
\hline Datasheets & $10 \Omega \pm 5 \%$ & $4.5 \mathrm{mH} \pm 3 \%$ & $12 \mu \mathrm{F} \pm 10 \%$ \\
\hline $\begin{array}{l}\text { Measured with } \\
\text { Frequency } \\
\text { Response }\end{array}$ & $10.53 \Omega \pm$ & $4.61 \mathrm{mH} \pm$ & $\begin{array}{l}11.57 \mu \mathrm{F} \pm \\
\text { Analyzer }\end{array}$ \\
\hline Identified with & $10.87 \Omega \pm$ & $0.7 \%$ & $0.7 \%$ \\
WSI technique & $11.7 \%$ & $16.5 \%$ & $11.93 \mu \mathrm{FH} \pm$ \\
\hline
\end{tabular}

15000 online measurements) and compared with the measured values given by the frequency analyzer from Venable Instruments (Table III). Results show that the WSI values are in line with those provided by the frequency analyzer and the datasheets of the components (a slightly larger resistance has been found, but this can be given by additional contact resistances). Table III also shows that the uncertainty of the online WSI technique is heavily affected by the contribution of all the possible uncertainties components (here including also phase-angles uncertainties, that have not been considered previously, which can be significant at high frequencies, for example due to time jitter and the sensing instruments) and thus it results in higher values.

It is worth noting that the obtained uncertainty values are in any case a tradeoff with the chosen identification process, which has been designed to provide fast responses. The complete identification process of the results in Figs. 11-12 takes $110 \mathrm{~ms}$, of which $100 \mathrm{~ms}$ are the chosen acquisition time window and the remaining $10 \mathrm{~ms}$ are needed for the impedance calculation (DFT and fitting routine).

\section{B. PHiL improvements via online WSI technique}

This subsection presents the experimental results of the application of the online WSI technique to the PHiL experiment. The governing question is whether the updating of the Damping Impedance $Z^{*}$ of the DIM IA results in improvements in accuracy and stability of PHiL experiment, thus validating (3) and the equations in Table I. Moreover, it is discussed how the accuracy of the DUT impedance identification given by the WSI technique impacts the stability and accuracy of the PHiL experiment. The ROS is modeled as an RL impedance with $10 \Omega$ and $5 \mathrm{mH}$. The results for the DUT configured as RL load were presented in [7], the results for the RLC load are presented in this paper.

Two cases, both using the DIM IA, but with different Damping Impedances are compared to analyze the stability margins improvement. The first case uses a rough estimate of the DUT impedance, i.e. purely resistive $5 \Omega$. This Damping Impedance guarantees the stable steady-state operating point needed to start up with the PHiL experiment. Then, the DUT impedance is identified to be an RLC load with $\mathrm{R}_{\mathrm{WSI}}=11.04 \Omega$, $\mathrm{L}_{\mathrm{WSI}}=4.40 \mathrm{mH}$, and $\mathrm{C}_{\mathrm{WSI}}=11.99 \mu \mathrm{F}$, configured as shown in Fig. 9. As last step, the damping impedance $Z^{*}$ is updated with these values.

\section{1) Frequency Domain - Stability}

A frequency response analyzer, i.e. a Model 350c from Venable Instruments, is used to plot the Nyquist diagram of the PHiL loop gain via loop injection technique [24]. The open loop gains of the PHiL setup without WSI (blue) and with WSI (red) are plotted in Fig. 13. The stability margins for both systems are included in cyan (phase margin) and yellow (gain margin). Both systems exhibit closed loop stability. Without WSI (blue) the

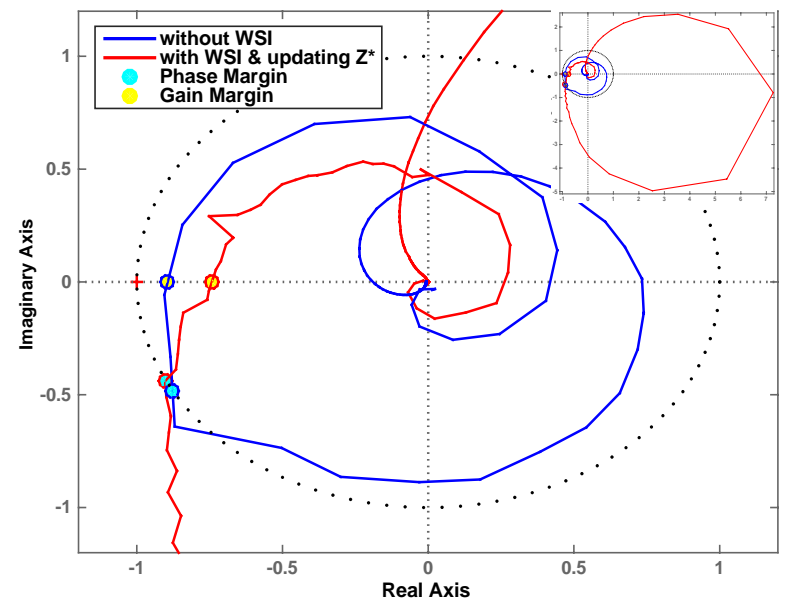

Fig. 13. Nyquist plot of the open loop gain of experimental PHiL setup comparing stability margins without (blue) and with WSI (red).

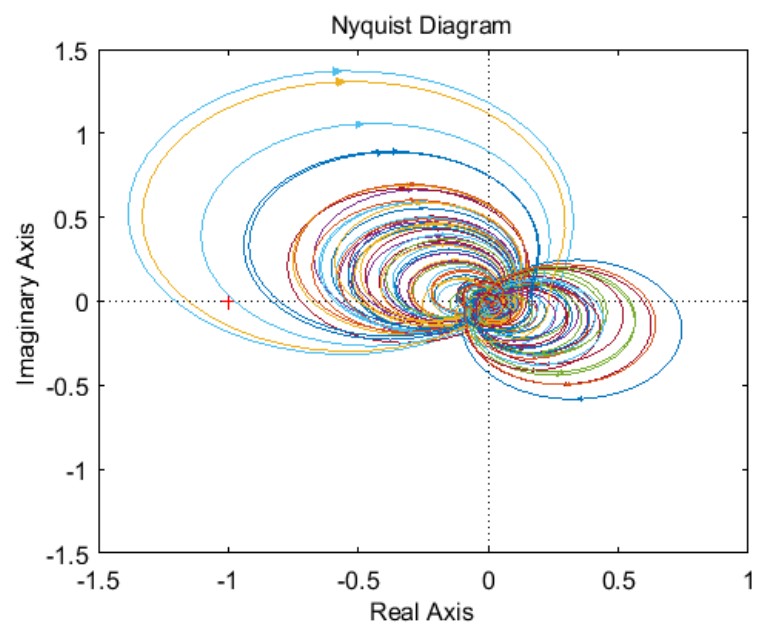

Fig. 14. Nyquist plot of the open loop gain with WSI parameters affected by large uncertainty. 
TABLE IV.

STABILITY EVALUATION OF PHIL EXPERIMENT WITH RLC LOAD, COMPARING WITH AND WITHOUT WSI.

\begin{tabular}{|c|c|c|}
\hline & Without WSI & With WSI \\
\hline Phase Margin & $28.9^{\circ}$ & $26.0^{\circ}$ \\
\hline Gain Margin & $0.98 \mathrm{~dB}$ & $2.58 \mathrm{~dB}$ \\
\hline
\end{tabular}

PHiL setup displays a phase margin of $28.9^{\circ}$ with a gain margin of roughly $1 \mathrm{~dB}$. For the PHiL system with updated Damping Impedance (red), the first thing that becomes apparent is the large circle spanned by its open loop gain. To illustrate the complete curve, a zoomed-out graph is displayed on the top right corner of Fig. 13. The reason for this large amplitude is that the impedances of ROS and DUT form a resonant circuit. In the time domain, this becomes apparent by an oscillatory transient behavior. The stability margins for both PHiL systems, without and with WSI, are listed in Table IV. By updating the Damping Impedance, the gain margin increases while the phase margin remains almost unchanged.

While both the previous cases were stable, possible issues can arise in case of large uncertainties in the identified impedances. Fig. 14 shows, as an example, the off-line simulation results obtained for the open loop gain when considering the identification of the impedance parameters affected by a large uncertainty. To this purpose 100 tests have been performed by randomly extracting the impedance parameters to simulate a $40 \%$ uncertainty. Results show that, in this scenario, such an uncertainty could bring to cases of instability in the system. This emphasizes the importance of having an accurate identification of the DUT impedance and of having a suitable characterization of the associated uncertainties in order to avoid possible stability issues during the PHiL simulation. In the presented scenario, for example, an accurate identification of the impedance parameters is mandatory to ensure the stability of the PHiL simulation.

\section{2) Time Domain-Accuracy}

The controlled voltage source in the ROS is ramped up, such that the DUT voltage rises with a slope of $50 \mathrm{~V} / \mathrm{ms}$ from $60 \mathrm{~V}$ to $110 \mathrm{~V}$. Voltage and current waveforms on the ROS side and DUT side are compared during the transients to the equivalent ones of the NCS (the reference) running in the same simulation environment.

For the case without WSI, the voltage (top) and current (bottom) waveforms of the NCS (black), ROS (red), and DUT (blue) are depicted in Fig. 15. The voltage and current waveform of the NCS exhibit oscillations, which neither ROS nor DUT can correctly mimic since the Damping Impedance is purely resistive and therefore does not show the same transient behavior as the RLC load. The limited bandwidth of the power amplifier is apparent in both voltage and current waveforms as the blue curve trails the ROS with a delay of approximately 500 $\mu \mathrm{s}$. Further, the large overshoot in current in the ROS (red) is due to the inaccurate Damping Impedance value.

After the WSI is performed and the Damping Impedance is updated, the same voltage ramp is applied again. The results depicted in Fig. 16 show a significant improvement in the

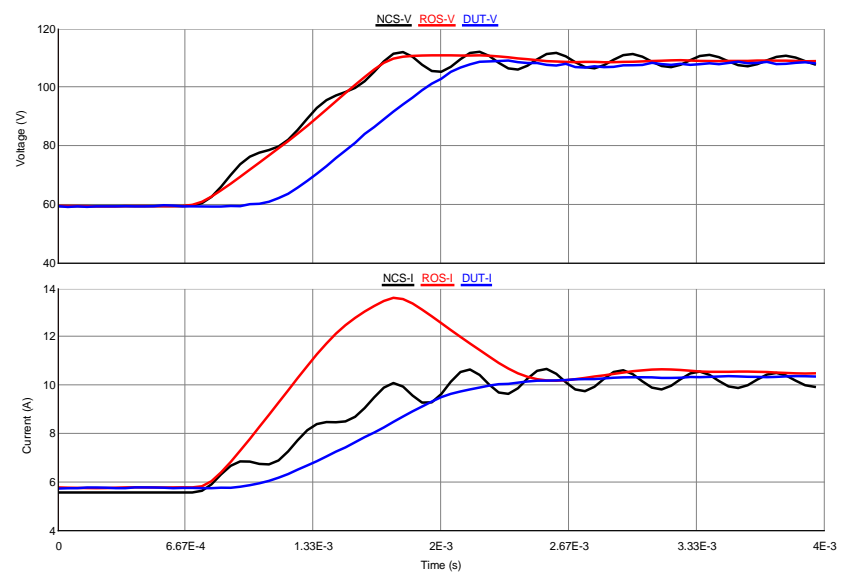

Fig. 15. Voltage ramp-up with initial resistive $5 \Omega$ Damping Impedance $Z^{*}$ comparing voltage (top) and current (bottom) waveforms of NCS (black), ROS (red), and DUT (blue).

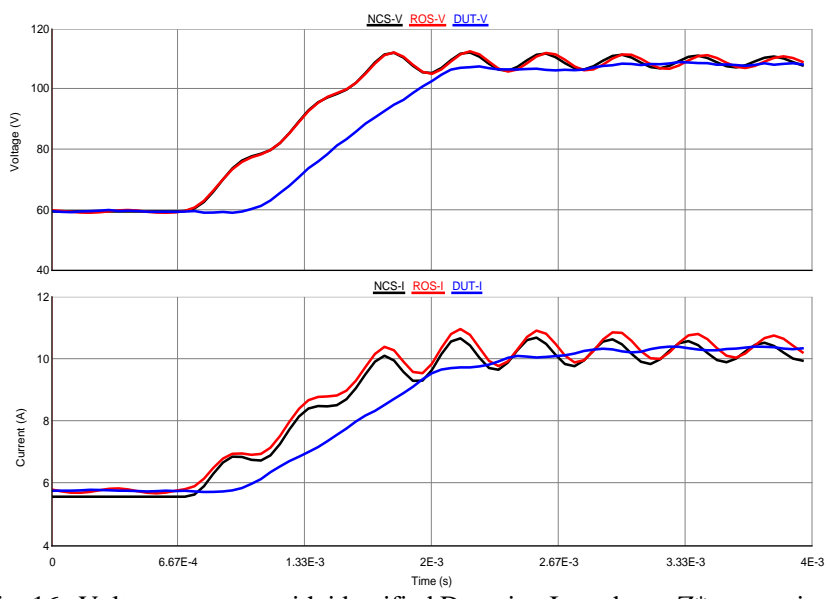

Fig. 16. Voltage ramp-up with identified Damping Impedance $Z^{*}$ comparing voltage (top) and current (bottom) waveforms of NCS (black), ROS (red), and DUT (blue).

dynamic accuracy. Both the voltage and current waveforms on the ROS side accurately follow the oscillatory transient of the NCS. On the DUT side, again, the limited bandwidth of the power amplifier prevents from tracking this oscillatory behavior. These two results are also predicted by the equations in Table I.

To assess the accuracy, the Root Mean Square Error (RMSE) is used in (8) as figure of merit. It quantifies the deviation between two waveforms in one single figure allowing a direct comparison between the system without WSI and with WSI.

$$
R M S E_{N C S R O S}=\sqrt{\frac{\sum_{t=1}^{n}\left(V_{N C S, t}-V_{R O S, t}\right)^{2}}{n}}
$$

Table $\mathrm{V}$ quantifies the deviations of the voltage on the ROS and DUT sides to those of the NCS for both cases without and

TABLE V.

ACCURACY EVALUATION COMPARING NCS AND PHIL (ROS AND DUT) WITHOUT AND WITH WSI

\begin{tabular}{|c|c|c|c|c|}
\hline & \multicolumn{2}{|c|}{ Without WSI } & \multicolumn{2}{c|}{ With WSI } \\
\cline { 2 - 5 } & ROS & DUT & ROS & DUT \\
\hline $\begin{array}{c}\text { RMSE } \\
\text { (to NCS) }\end{array}$ & $2.2 \mathrm{~V}$ & $10.5 \mathrm{~V}$ & $0.7 \mathrm{~V}$ & $9.7 \mathrm{~V}$ \\
\hline
\end{tabular}




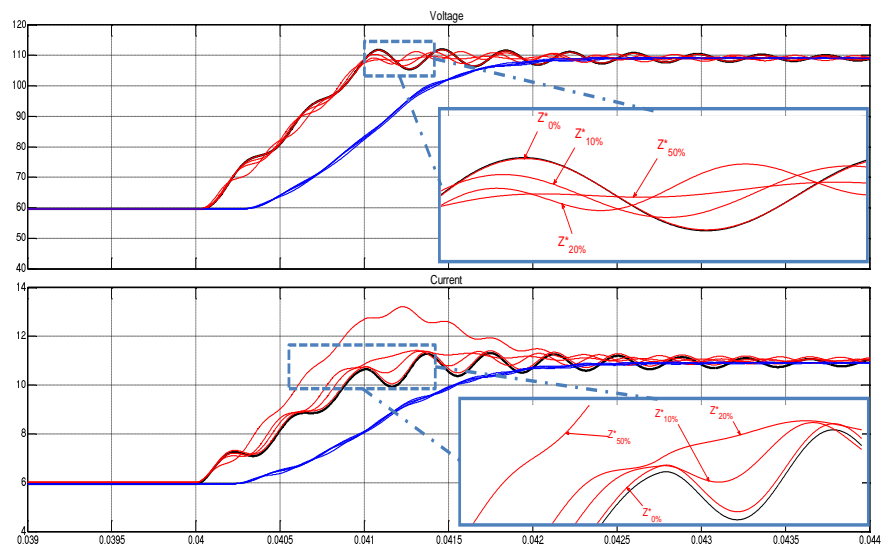

Fig. 17. Voltage ramp-up for four sub-optimal Damping Impedances $Z^{*}$ comparing voltage (top) and current (bottom) waveforms of NCS (black), ROS (red), and DUT (blue).

with WSI by using (8). The RMSE on the ROS side improves dramatically from $2.2 \mathrm{~V}$ (without WSI) to as little as $0.7 \mathrm{~V}$ deviation with WSI. On the DUT side, the RMSE reduces by $0.8 \mathrm{~V}$ when updating the Damping Impedance.

To evaluate how the accuracy of the DUT impedance identification given by the WSI technique affects the accuracy of the PHiL experiment, simulations were run by considering increasing errors in the identified impedance. Fig. 17 shows the effect of four sub-optimal Damping Impedances whose RLC parameters are considered with negative errors equals to $0 \%$, $10 \%, 20 \%$, and 50\%. The same voltage ramp-up of Figs. 15-16 is used. In line with the previous results, it is shown that only a very accurate identification of the DUT impedance (the $Z_{0 \%}^{*}$ case in zoomed-in sections of Fig. 17) can guarantee voltage and current of the PHiL system on the simulation side to accurately follow the resonant oscillations of the NCS. Instead, in this scenario, voltages and currents on the DUT side of the PHiL system are not affected by these sub-optimal Damping Impedances in an appreciable way.

In conclusion, some considerations are made in regards to the possible applications of the online WSI technique coupled to a PHiL setup with DIM IA to test power hardware such as loads, converters, generators and protective devices, connected to complex power system models running in the real-time simulator. The case of a real static synchronous compensator (STATCOM) to provide voltage regulation through reactive power injection to the grid it is connected to is considered as an example. When building the PHiL system to test such an application, the real STATCOM is the DUT and it is connected to the grid model running in the real-time simulator through the IA and power amplifier. When testing such an application in PHiL with DIM IA, inaccurate knowledge of the STATCOM impedance over all operative conditions may lead to inaccurate results or even to instability. Therefore, to cope with this limitation, the online WSI technique can be particularly useful to provide the stability and accuracy improvements described thoroughly in this paper. Notice that the accuracy improvements described in this paper are only on the simulation side given the relatively low bandwidth of the power amplifier. If a power amplifier with higher bandwidth is used in the PHiL setup, the method of the online WSI technique coupled to a
PHiL with DIM IA will provide accuracy improvements on the DUT side as well, as reported on the right column of Table I.

\section{CONCLUSIONS}

This paper presented the accuracy and stability improvements achievable for PHiL simulations interfaced to a DIM IA employing online WSI technique. The implementation details of the WSI technique were presented, together with the hardware and software set-up needed for its integration into the PHiL platform. A qualitative evaluation of the different contributions to the overall uncertainty of the WSI chain has been provided and tests have been performed to assess the impact of input uncertainties on the post-processing routines. Tests demonstrated the capability of the WSI technique to correctly identify the impedance parameters by using very small time windows and without severe consequences on the uncertainty performance. The benefits provided by the presented technique are shown in the context of PHiL simulations, where identified impedances are used to online update the Damping Impedance used in the real-time simulator. The performed test cases using an RLC impedance as DUT in the PHiL system showed the clear improvements achievable in terms of both stability and accuracy allowed by the proposed implementation of the WSI technique.

\section{REFERENCES}

[1] B. Miao, R. Zane, and D. Maksimovic, "System Identification of Power Converters With Digital Control Through Cross-Correlation Methods," IEEE Trans. Power Electron, vol. 20, no. 5, pp. 1093-1099, 2005

[2] A. Barkley and E. Santi, "Online Monitoring of Network Impedances Using Digital Network Analyzer Techniques," Applied Power Electronics Conference and Exposition, 2009. APEC 2009. Twenty-Fourth Annual IEEE, Washington, DC, 2009, pp. 440-446

[3] D. Martin, E. Santi, and A. Barkley, "Wide bandwidth system identification of $\mathrm{AC}$ system impedances by applying pertubations to an existing converter," Energy Conversion Congress and Exposition (ECCE), 2011 IEEE, vol., no., pp.2549,2556, 17-22 Sept. 2011

[4] D. Martin, I. Nam, J. Siegers, and E. Santi, "Wide bandwidth three-phase impedance identification using existing power electronics inverter," Applied Power Electronics Conference and Exposition (APEC), 2013 Twenty-Eighth Annual IEEE, vol., no., pp. 334,341, 17-21 March 2013

[5] A. Riccobono, S. K. A. Naqvi, A. Monti, T. Caldognetto, J. Siegers, and E. Santi, "Online wideband identification of single-phase AC power grid impedances using an existing grid-tied power electronic inverter," Power Electronics for Distributed Generation Systems (PEDG), 2015 IEEE 6th International Symposium on, vol., no., pp.1-8, 22-25 June 2015

[6] A. Riccobono, E. Liegmann, A. Monti, F. Castelli Dezza, J. Siegers and E. Santi, "Online wideband identification of three-phase AC power grid impedances using an existing grid-tied power electronic inverter," 2016 IEEE 17th Workshop on Control and Modeling for Power Electronics (COMPEL), Trondheim, 2016, pp. 1-8

[7] E. Liegmann, A. Riccobono, and A. Monti, "Wideband Identification of Impedance to improve Accuracy and Stability of Power-Hardware-in-theLoop Simulations," Applied Measurements for Power Systems (AMPS), 2016 IEEE International Workshop on, 28-30 Sept. 2016

[8] Z. Staroszczyk, "A method for real-time, wide-band identification of the source impedance in power systems," in IEEE Trans. on Instrumentation and Measurement, vol. 54, no. 1, pp. 377-385, Feb. 2005

[9] R. Stiegler, J. Meyer, P. Schegner, and D. Chakravorty, "Measurement of network harmonic impedance in presence of electronic equipment," Applied Measurements for Power Systems (AMPS), 2015 IEEE International Workshop on, vol., no., pp. 49-54, 23-25 Sept. 2015

[10] T. T. Do, M. Jordan, H. Langkowski, and D. Schulz, " Novel Grid Impedance Measurement Setups in Electrical Power Systems," Applied 
Measurements for Power Systems (AMPS), 2016 IEEE International Workshop on, 28-30 Sept. 2016

[11] D. Serfontein, J. Rens, G. Botha, and J. Desmet, "Improved Event Based Method for Harmonic Impedance Assessment," Applied Measurements for Power Systems (AMPS), 2016 IEEE International Workshop on, 2830 Sept. 2016

[12] D. Serfontein, J. Rens, G. Botha and J. Desmet, "Continuous Event-Based Harmonic Impedance Assessment Using Online Measurements," in IEEE Transactions on Instrumentation and Measurement, vol. 65, no. 10, pp. 2214-2220, Oct. 2016

[13] BIPM, IEC, IFCC, ILAC, ISO, IUPAC, IUPAP, OIML; Evaluation of Measurement Data - Guide to the Expression of Uncertainty in Measurement , 2008

[14] N. Locci, C. Muscas, E. Ghiani, Evaluation of uncertainty in measurements based on digitized data, Elsevier, Measurement 32 (2002) 265-272

[15] G. Betta, C. Liguori, A. Pietrosanto, Propagation of uncertainty in a discrete Fourier transform algorithm, Elsevier, Measurement 27 (2000) 231-239

[16] C. S. Edrington, M. Steurer, J. Langston, T. El-Mezyani and K. Schoder, "Role of Power Hardware in the Loop in Modeling and Simulation for Experimentation in Power and Energy Systems," in Proc. IEEE, vol. 103, no. 12, pp. 2401-2409, Dec. 2015

[17] S. Pijpers, N. Blaauwbroek, P. Nguyen, and H. Slootweg, "Data Acquisition and Processing for Power Hardware in the Loop Simulations of LV Distribution Feeders," Applied Measurements for Power Systems (AMPS), 2016 IEEE International Workshop on, 28-30 Sept. 2016

[18] W. Ren, M. Steurer and T. L. Baldwin, "Improve the Stability and the Accuracy of Power Hardware-in-the-Loop Simulation by Selecting Appropriate Interface Algorithms," Industrial \& Commercial Power Systems Technical Conference, 2007. ICPS 2007. IEEE/IAS, Edmonton, Alta., 2007, pp. 1-7

[19] J. Siegers and E. Santi, "Improved power hardware-in-the-loop interface algorithm using wideband system identification," Applied Power Electronics Conference and Exposition (APEC), 2014 Twenty-Ninth Annual IEEE, Fort Worth, TX, 2014, pp. 1198-1204

[20] J. Siegers, H. L. Ginn and E. Santi, "Stability and accuracy considerations in the design and implementation of a kilowatt-scale DC power hardwarein-the-loop platform," Energy Conversion Congress and Exposition (ECCE), 2014 IEEE, Pittsburgh, PA, 2014, pp. 1126-1133

[21] T. Hatakeyama, A. Riccobono and A. Monti, "Stability and accuracy analysis of power hardware in the loop system with different interface algorithms," 2016 IEEE 17th Workshop on Control and Modeling for Power Electronics (COMPEL), Trondheim, 2016, pp. 1-8

[22] E. Liegmann, A. Riccobono, A. Monti, T. Hatakeyama, "A Flexible Power Simulator (FlePS): Power Interface for Power Hardware in the Loop applications - A Performance Evaluation," Power and Energy Student Summit 2016 (PESS 2016) Proc.eedings of the Conference on, Aachen, January 19-20 2016, S_E.01

[23] A. Benigni, A. Helmedag, A. M. E. Abdalrahman, G. Pilatowicz and A. Monti, "FlePS: A power interface for Power Hardware In the Loop," Power Electronics and Applications (EPE 2011), Proceedings of the 2011-14th European Conference on, Birmingham, 2011, pp. 1-10

[24] R. W. Erickson and D. Maksimovic, Fundamentals of Power Electronics, 2nd ed. Boston, MA:Kluwer, 2001, pp. 362-368

[25] E. C. Levy, "Complex-curve fitting," in IRE Transactions on Automatic Control, vol. AC-4, no. 1, pp. 37-43, May 1959 